\title{
The Paramount Importance of Technical Equivalence Assessment for Active Substances in Crop Protection and Biocidal Products
}

\begin{abstract}
Hanna Skarpos*
This article outlines the importance of assessing the technical equivalence regarding the chemical composition and hazard profile for different new sources of an active substance compared to the reference source of the same active substance used for the initial risk assessement. The legal provisions of past directives and two current regulations as last amended, Regulation (EC) No 1107/2009 concerning the placing of plant protection products on the market and Regulation (EU) No 528/2012 concerning the placing on the market and use of biocidal products, are addressed in detail. Moreover, the harmonised procedures for assessing technical equivalence of different sources, based on the information presented in different supporting guidelines, are portrayed and discussed. Following the presentation of all legal provisions, a two-tiered approach as proposed for assessing the equivalence of different sources of active substances is presented in detail.
\end{abstract}

\section{Introduction}

The synthesis of active substances included in formulations of plant protection products and biocidal products is a complex process consisting of different reactions. The chemical and physical manipulation of processes involved in the synthesis must be repeatable and reliable to ensure the same substance identity profile of the produced technical material. The producer of the active substance manufactures the active substance is choosing the relevant starting materials, solvents, equipments, chemical conditions, purification procedures, equipment cleaning protocols, etc. The compilation of the synthesis-related data of an active substance is only a small part, however a very essential one, of the long and extensive evaluation process of the active substance approval, in which different parties are actively involved: the European Food Safety Authority (EFSA), European Chemical Agency (ECHA), the European Commis-

DOI: $10.21552 / \mathrm{icrl} / 2019 / 1 / 5$

* Dr. Hanna Skarpos is a scientific expert / regulatory chemist at knoell Germany. She has many years experience in preparation of different technical equivalences. She would like to express her appreciation to her colleagues Dr. Daniela Fruth, Dr. José João Dias Carvalho and Dr. Michael Cleuvers for their support. For correspondence: <hskarpos@knoell.de> sion (EC) and additionally all concerned Member States.

During the complex approval process of an active substance the specifications, encompassing the minimum purity of the active substance and the maximum content of impurities derived from the reference(s) source(s), are set. The content of the active substance and impurities have to be deducible considering the results obtained from the certificate of analysis of five representative batches and/or the associated quality control data. The specification derived from the approval procedure of the active substance is defined as the 'reference specification' for that active substance at the EU level, and it is valid for the entire period of the approval. Yet very often, the synthesis of the active substance needs to be modified or the location of the manufacturing site changes. Thus, an evaluation needs to be (re)conducted to show that the specification derived from the new source is equivalent to the reference source, ie a technical equivalence assessment needs to be performed.

Even if the purpose and use of an active substance in plant protection products or biocidal products may be different, and different regulatory frameworks apply, the approach for the evaluation of technical equivalence is identical, in both cases based on a tiered approach. During this two-tiered approach, 
the focus is on the purity level of the active substance and its impurities. Since the increase of impurities in the new source (in comparison to the reference source) or the presence of new impurities in the new source can affect the risk assessement carried out during the approval of active substance, the evaluation of technical equivalence is considered of paramount importance. The safety and efficacy assessments performed for the approval of the active substance are only valid for the original sources, thus any 'new' sources or manufacturing plants need to prove its equivalence to the original reference source.

\section{The Principles of Technical Equivalence in the Scope of Past and Current Legislations}

\section{View on Plant Protection Products Legislations}

In retrospect, the former Council Directive 91/414/EEC of 15 July $1991^{1}$ concerning the placing of plant protection products on the market (repealed by Regulation (EC) No 1107/2009 on 14/06/2011), did not provide a clear guidance for the assessment of technical equivalence. The specification's requirements referring to the inclusion of any active substance into Annex I of the former Directive (Article 5) were limited to the following: the minimum degree of purity of the active substance, the nature and maximum content of certain impurities, type of preparation and manner of use.

For the purpose of evaluating the technical specification and the impurities' profile of the active substance for compliance with the specification established, allowing for its Annex I inclusion, the European Commission had published a working Guidance Document on the Assessment of the Equivalence of Technical Equivalence of Substance Regulated under Council Directive 91/414/EEC, namely SANCO/10597/2003 -rev. 8.1 May 2009) in 2009, which was elaborated in cooperation with the Member States. The intention of the corresponding guidance was not to 'produce legally binding effects (...) not to prejudice any measure taken by a Member State within the implementation prerogatives under Annex II, III and VI of Council Directive 91/414/EEC (...) not preclude the possibility that the European Court of Justice may give one or another provision direct effect in Member States, ${ }^{2}$ but to present a harmonised procedure for assessing the equivalence of different sources of technical material versus the reference source. Notwithstanding, the guidance should assist the Rapporteur, ie the Member State (MS) performing the assessement on behalf of all concerned MS, when assessing the equivalence of different sources during the evaluation for Annex I inclusion. It should also guide the national authorities during the registration process when new sources would need to be included. It is important to note that the afore-mentioned document neither address active substances present in plant protection products that are microorganisms nor active substances that have poorly defined chemical compositions/mixtures, eg plant extracts, animal products and their derivatives. In summary, the document provided guidance for synthetic chemicals with a welldefined identity.

Regulation (EC) No 1107/2009 came into effect in June 2011. ${ }^{3}$ The Regulation brought along a more detailed view on the procedure to perform technical equivalence assessment of active substances to be included in plant protection products.

The provisions referring to the assessment of equivalence of different sources, or the same source after a change of the manufacturing process or manufacturing plant location, is stipulated in Article 38 of Regulation (EC) No 1107/2009. The content of article 38 is clear: the Member State acting as rapporteur for the active substance should assess the equivalence. Additionally, the other Member State(s) examining the application for the authorisation or amendment of an authorisation of plant protection products can access the application for technical equivalence in parallel after having agreed the details with the rapporteur for the active substance.

According to Article 38, to assess the equivalence during the evaluation process, either the rapporteur Member State (RMS) or designated Member State (DMS) is obliged to prepare the respective report on

1 Council Directive of 15 July 1991 concerning the placing of plant protection products on the market (91 / 414 / EEC).

2 Guidance Document on the Assessment of the Equivalence of Technical Materials of Substances Regualted under Regulation (EC) No 1107/2009, SANCO/10597/2003 - rev 8.1 [May 2009].

3 Regulation (EC) No 1107/2009 of The European Parliament and of the Council of 21 October 2009 concerning the placing of plant protection products on the market and repealing Council Directives 79/117/EEC and 91/414/EEC. 
equivalence within 60 days from the date of receiving the application. Any disagreement between Member States' opinions should be communicated to the applicant and the European Commission. The applicant is allowed to submit his comments and the time to reach an agreement is set at 45 days. The Commission can ask the Authority (EFSA) for either an opinion or scientific and technical assistance, which shall be provided within 3 months. Even if the timelines established in the Regulation for the assessment are relatively short, in practice the assessment phase is often taking much longer until the technical equivalence is finally granted.

It is important to note here that any changes concerning the sources of the technical material after the authorisation of a plant protection product are dealt with by Article 45(2) of Regulation 1107/2009, ie with an amendment of the formulated product authorsation at Member State level.

In response to the implementation of Regulation $1107 / 2009$, a new version of the working Guidance Document on the Assessment of the Equivalence of Technical Equivalence of Substance Regulated under Regulation (EC) No 1107/2009 (SANCO/10597/2003 - rev. 10.1, 13 July 2012) had been prepared by the European Commission to replace the previous version from 2009. This new adopted Guidance Document has established a harmonised procedure for assessing the equivalence between different sources of technical material according to Art. 38 of the Regulation 1107/2009 following the two-tiered approach presented originally in version 8.1 (dated May 2009) ${ }^{4}$.

\section{View on Biocidal Products legislations}

In a retrospective view, under the former Biocidal Products Directive 98/8/EC (BPD), the technical equivalence was assessed (in similarity to plant protection products) by the Member State Competent

4 Guidance Document on the Assessment of the Equivalence of Technical Materials of Substances Regualted under Regulation (EC) No 1107/2009, SANCO/10597/2003 - rev 10.1 [July 2012].

5 Directive 98/8/EC of the European Parliament and of the Council of 16 February 1998 concerning the placing of biocidal products on the market [1998] L 123/1.

6 Technical Notes for Guidance on the assessment of technical equivalence of substances regulated under Directive 98/8/EC.

7 Regulation (EU) No 528/2012 of the European Parliament and of the Council of 22 May 2012 concerning the making available on the market and use of biocidal products.
Authority (MSCA). In the corresponding Directive, no information was provided on the evaluation of the technical equivalence, however pursuant to Article 33 of the Directive: the Commission (...) shall draw up technical notes for guidance to facilitate the dayto-day implementation of this Directive. ${ }^{5}$ Consequently, during the $29^{\text {th }}$ meeting of representatives of Members States Competent Authorities for the implementation of Directive 98/8/EC concerning the placing of biocidal products on the market, which was held from 28 - 30 May 2008, Technical Notes for Guidance (TNsG) on the assessment of technical equivalence of substances regulated under Directive 98/8/EC were adopted. It is important to note that this document is conceived as a working document and did not intend to produce legally binding effects and by its nature (...) not prejudice any measure taken by a Member State within the implementation prerogatives of Directive 98/8/EC, nor any case law developed with regard to this provision. ${ }^{6}$

The Biocidal Product directive (Regulation (EU) No 528/2012) was published in 2012 and became applicable on the $1^{\text {st }}$ of September, 2013. The Regulation provides a centralised provision for the assessment of technical equivalence of active substances. Technical equivalence is defined in the BPR in Article $3(1)(w)$ : 'technical equivalence' means similarity, as regards the chemical composition and hazard profile, of a substance produced either from a source different to the reference source, or from the reference source but following a change to the manufacturing process and/or manufacturing location, compared to the substance of the reference source in respect of which the initial risk assessment was carried out, as established in Article 54."

Article 54 of the BPR provided the legal basis is, setting out the procedure for the assessment of technical equivalence applications and assigns the responsibility to the European Chemical Agency (ECHA). In the first step, the applicant (person seeking a technical equivalence for the active substance) submits the required data to Agency via $\mathrm{R}_{4} \mathrm{BP}_{3}$. ECHA informs the applicant about the fee to be paid (no payment of the fee within the period of 30 days can cause the rejection of the application). In general and as stated in Article 54(4), the Agency has 90 days to take a decision on the technical equivalence application. Considering that additional information may need to be submitted, the 90-day period mentioned shall be suspended from the date of is- 
sue of the request until the information is received. The suspension however shall not exceed 180 days except where justified by the nature of the data requested or in exceptional circumstances. It is important to mention, that the Agency can consult the competent authority of the Member State, which acted as the evaluating competent authority for the evaluation of the active substance as provided by Article $54(6)$.

Regulation (EU) No 837/2013 (2013) amended Annex III of the BPR as regards to the information requirements for authorisation of biocidal products (Annex III refers to information requirements for biocidal products). In this amendment, the necessity to provide evidence when requesting technical equivalence for the active substances in the biocidal product is given. In detail: where the biocidal product contains an active substance that has been manufactured in locations or according to processes or from starting materials other than those of the active substance evaluated for the purpose of approval pursuant to Article 9 of this Regulation, evidence has to be provided that technical equivalence has been established in accordance with Article 54 of this Regulation or has been established, following an evaluation having started before 1 September 2013, by a competent authority designated in accordance with Article 26 of Directive 98/8/EC. ${ }^{8}$

In response to the new regulation and the associated amendments, ECHA published the first version of the Guidance Document on applications for technical equivalence in August 2013. The current version 2.0 of the document published in July 2018 was fully revised to address a series of important issues arising from the current status of scientific knowledge and assessment procedure (and obsolete information has been removed).

\section{Assessment of Technical Equivalence - Main Principles}

The intention and general principle of technical equivalence assessment is the determination of the similarity of its chemical compositions and hazard profiles. The initial risk assessment of the active substance is based on the reference source, or a reference specification encompassing the profile of several sources. In the documentation referring to the approval of the active substance, only one reference specification can exist for one active substance. In addition, in case the approval of the active substance has been applied for by different applicants, as Task Forces or Consortiums, a single reference specification needs to be set. The source for which the assessment of technical equivalence needs to be conducted is called the new or alternative source as provided in the legal texts and other supporting documents.

The main prerequisite to be considered for examination of technical equivalence is the identity of the active substance: the active substance from the new or alternative source needs to have the same identity as the active substance from the reference source(s), otherwise, the assessment for technical equivalence cannot be conducted. More precise information on substance identity can be found in the Guidance for identification and naming of substances under REACH and CLP. ${ }^{9}$ As indicated by ECHA in the Guidance on applications for technical equivalence, any uncertainties should be clarified by contacting the Agency via the helpdesk. The more complex case for UVCB substances (Unknown or Variable composition, Complex reaction products or Biological materials) and microorganism are addressed separately toward the end of this article.

As already mentioned above, a technical equivalence to the reference specification will be needed if the active substance originates from a different manufacturer, there is a change of the manufacturing location, the manufacturing process (change in solvents, reactants, equipment, purification process, and/or quality of starting materials). Because of the scenarios listed above the resulting minimum purity of the active substance, based on the results obtained from five batch analysis study and/or quality control data, can be lower than the purity set during approval of the active substance (non equivalence of the source). Also, higher levels of impurities or completely new impurities could be present due to the above-mentioned changes, thus resulting in a nonequivalence of the new source.

The application for the assessment of technical equivalence can be submitted by any applicant (eg

8 Commission Delegated Regualtion (EU) No 837/2013 of 25 June 2013 amended Annex III to Regulation (EU) No 528/2012 of the European Parliament and of the Council as regards the information requirements for authorisation of biocidal products.

9 Guidance for identification and naming of substances under REACH and CLP May 2017 Version 2.1. 
supplier of the active substance, formulator of enduser products, new manufactor), whose aim is to present a new source of the active substance, a change in location of the manufacturing plant, changes in the manufacturing processes or simply switching the production from the pilot scale to an industrial scale.

\section{Tiered Approach}

In case of a plant protection product the rapporteur Member State (RMS) conducts the technical equivalence assessment in a two-tiered approach (in case of a biocidal product, ECHA will do it). Applicants submitting the application for technical equivalence should be aware that the requirements for Tier I (chemistry) differ from the requirements for the Tier II assessment (toxicology and ecotoxicology). Tier II will be necessary only if the technical equivalence cannot be shown at Tier I level (chemical equivalence).

\section{Tier I - Evaluation of Equivalence of the Technical Material (Chemistry Assessment)}

The evaluation of Tier I (chemistry) consists mainly in comparing the chemical compositions of technical grade active substances originating from different sources, based on the five batch analysis study. At least five representative batches from current industrial scale production of the active substance shall be analyzed for content of pure active substance, impurities, additives and each further component other than additives, as appropriate. ${ }^{10}$ The outcome of the corresponding study allows specifying the minimum purity of the pure active substance and the maximum content of all identified impurities. The ana-

10 Commission Regulation (EU) 283/2013 setting out the data requirements for active substances, in accordance with Regulation (EC) No 1107/2009 of the European Parliament and of the Council concerning the placing of plant protection products on the market of 1 March 2013, L 93/1.

11 Technical Material and Preparations: Guidance for generating and reporting methods of analysis in support of pre- and post-registration data requirements for Annex II (part A, Section 4) and Annex III (part A, Section 5) of Directive 91/414, SANCO/3030/99 rev.4 11/07/00. lytical method(s) used needs to be validated with regards to linearity, specificity, repeatability and accuracy following SANCO 3030/99 rev. 4.1 (11.07.2000) guideline. ${ }^{11}$

As a rule of thumb, the five-batches analyzed should not be older than five years. In certain cases where the batches data of new source are slightly older than five years, the applicant can provide supporting data, the so-called Quality Control (QC) data, if technical equivalence is sought for a change in manufacturing location. In general, the QC data can be submitted as supporting data for different refinement purposes, as for example modification of minimum purity of the active substance or maximum levels of impurities. However, it is important to point out here, that QC cannot replace the requirement to provide five batch analytical data.

The requirements on analytical data to be provided can differ slightly, depending on the scenarios under which the assessment of technical equivalence is prepared. For example, in case the applicant submits a technical equivalence application for a change from pilot to large scale production, data referring to the description of identity of the chemical substance, manufacturing processes, specification of purity of the active substance and identity of purity of active substance can be covered by an applicant statement without the need to generate extra data. Yet, there is a need for new analytical data if the large-scale production has brought about a change to the impurity profile or a different analytical procedure was implemented.

It is important to point out here that for the technical equivalence application Tier I under BPR, information on spectral data and optical activity is required but not for Tier I evaluation under Regulation $1107 / 2009$. The spectral data has to be submitted for one of the batches, considered to be representative for the current production.

After the evaluation process (comparison) of two sources, the new source is considered equivalent to the reference source if all the following conditions are met:

- The minimum degree of purity obtained with the alternative source is equal to or higher than the one obtained with the reference source.

- No new impurity or additive is present.

- The limit of each relevant impurity or additive is not exceeded. 


\begin{tabular}{|c|c|}
\hline $\begin{array}{l}\text { Certified limits of significant but non-relevant } \\
\text { impurities in the reference technical specifications }\end{array}$ & $\begin{array}{l}\text { Acceptable maximum increase in the new / } \\
\text { alternative source } \\
\text { (based on FAO Specification Manual) }\end{array}$ \\
\hline$\leq 6 \mathrm{~g} / \mathrm{kg}$ & $3 \mathrm{~g} / \mathrm{kg}$ \\
\hline$>6 \mathrm{~g} / \mathrm{kg}$ & $50 \%$ of the certified limit \\
\hline
\end{tabular}

Table 1: Acceptable Levels for Certified Limits of Non-relevant Impurities

- The limits of all significant but not relevant impu rities are not exceeded by more than the levels in dicated in Table $\mathbf{1}$ above.

It is noteworthy that the criteria specified in Table 1, which are based on the FAO Specification Manual, are only applicable if the evaluation of certified limits of impurities refers to impurities, which were present in the reference specification supporting the approval of the active substance.

Additional conditions for evaluation of a multiconstituent substance need to be considered: Technical equivalence can be granted, if each main constituent remains in the $10-80 \%$ range and the concentration of each main constituent does not deviate by more than $5 \%$ absolute or $10 \%$ relative, whichever is larger, as stated in Guidance on BPR (Guidance on applications for technical equivalence). ${ }^{12}$

Based on the conducted evaluations, a technical equivalence could be granted to the new source based on Tier 1 assessment (chemistry). Incase two sources are not technically equivalent based on this Tier 1 assessment, further evaluations with regards to toxicological and ecotoxicological hazard are necessary for the alternative source (Tier II assessment).

\section{Tier II - Evaluation of Equivalence of the Technical Material (Toxicology and Ecotoxicology Assessment)}

The objective of the Tier II assessment is to ensure that there is no unacceptable change in the (eco)toxicological hazard profile of the new source compared to the reference specification as a result of the change of the composition (eg presence of a new impurity). Thus the Tier II assessment needs to include a reasoned case, and if necessary also data, to show that the alternative source does not have more severe (eco)toxicological hazard properties than the reference specification, including bioaccumulation and environmental persistence.
The main approach of this evaluation is to show that the new impurities found in the new source do not present a higher risk for human health and the environment compared to the reference source.

Impurities for Tier II assessment can be categorised as follows:

- new impurity or additive;

- increased levels of relevant impurities or additives;

- increased levels of significant but non-relevant impurities;

- impurities of no toxicological concern;

- impurities of known toxicological concern;

- and new impurities of unknown toxicological concern or increased levels of significant but non-relevant impurities.

In general, Tier II information requirements should be provided to cover all impurities, including sometime new data and QSAR-generated information. However, for impurities for which (eco)toxicity is known to be low (eg water, certain inert materials, mineral salts) a justification explaining why no information was provided and that a Tier II assessment is not necessary is usually considered sufficient. The outcome of this first categorisation is quite simple: no further evaluation is required. The applicant can treat both sources as equivalent. The respective justification will have to be submitted either to ECHA for biocidal applications or to RMS (Rapporteur Member State) in the case of plant protection products applications.

In case an impurity of toxicological relevance was identified in the new source, the applicant needs to provide evidence that the presence of this impurity will not cause any significant changes to the toxico-

12 ECHA, 'Guidance on the Biocidal Products Regulation Volume V: Guidance on Applications for Technical Equivalence' (July 2018) $<$ https://echa.europa.eu/documents/10162/23036412/guidance _applications_technical_equivalence_en.pdf/18f72d37-98b6 -47c8-98bb-941 afeff6968> accessed 12 February 2019. 
logical profile of the active substance. Also in the case of a new impurity of unknown toxicological concern in the new source, suitable information should be provided. As indicated in SANCO/10597/2003 -rev. 10.1, the reliance should be placed on information that is already available, ie new testing should be avoided. Based on evidence demonstrating that the increased level of an impurity (or new impurity) will not have a significant adverse effect on the toxicity of the new source, the conclusion of equivalence of sources can be drawn. In case impurities are present in the alternative source leading to an increase of the toxicological hazard compared to the reference source, then a technical equivalence cannot be granted.

The assessment of the ecotoxicological equivalence is analogous to the toxicological one. The main objective of the evaluation is identification of new impurities, or higher impurity levels, with an impact on the ecotoxicological safety of the technical material. Based on the evidence showing that either the increased level of an existing impurity or new impurity will not have a significant adverse effect, the conclusion of sources' equivalence can be established. In case impurities are present in the alternative source, leading to an increase of the (eco)toxicological hazard a technical equivalence to the new source cannot be granted.

The corresponding Guidance Documents, the Guidance on the assement of the equivalence of technical materials of substances regulated under Regulation (EC) No 1107/2009 and Guidance on the Biocidal Products Regulation Volume V, Guidance on applications for technical equivalence, provide detailed instructions on the decision making processes and allow to correctly evaluate impurities in view of (eco)toxicological hazards.

\section{Tiered-approach for UVCB Substances}

In the above-mentioned Guidance on BPR Volume V published by ECHA in July 2018, a separated section is dedicated to the assessment of technical equivalence for substances of Unknown or Variable composition, Complex reaction products or Biological ma-

13 Steven Buchanan, 'Substance identity - UVCB substances' (Lead registrant workshop, 2 February 2012) <https://echa.europa.eu/ documents/10162/22816103/10_sb_siduvcb_d1_Irws_20120203 _en.pdf $>$ accessed 12 February $201 \overline{9}$. terials, also called UVCB substances. Because many plant extracts can be categorised as UVCB substances and are used as active substance in biocidal or plant protection products, the discussion of equivalence assessment for such substances needs to be included in the scope of this article.

The identification of such chemicals is not easy and sometimes not possible since the number of constituents is relatively large, the exact composition is unknown and the variability of its components is relatively large and poorly characterised. ${ }^{13}$ In general, the approach for the assessment of the technical equivalence follows the same principle as described for mono and multi-constituent active substances, namely the two-tiered approach. However, taking the different limitations arising from the complex and variable composition of UVCB substances into account, ECHA recommends considering directly preparation and submission of Tier II application without a prior Tier I assessment. The Tier I application can meet obstacles on the path of acceptance due to analytical limitations of the analysis (for example identification and quantification) of single constituents present in the complete composition of UVCB substances. Nonetheless, Tier I assessments presenting good results and arguments for technical equivalence can be considered acceptable on a caseby-case basis.

In order to grant technical equivalence in a Tier I Assessment, the following criteria need to be met as proposed by ECHA: both sources' compositions are fully accounted (5-batch analysis up to $98 \%$ closure), single chemical constituents of the UVCB substance are clearly defined (no presence of unknown and/or unspecific constituents), and finally the specifications arriving from both sources have defined ranges for concentrations or other physicochemical parameters, if relevant.

Similarly, to mono constituent active substances, the Tier II assessment of UVCB substances needs to be considered as an interactive approach. Some of the theoretical basis for the different approaches during Tier II assessment focus on effects assessment and are presented in the Guidance document in BPR (volume V). The Guidance document provides descriptions for the so-called known constituents' approach, block/fraction approach (grouping of constituents) and whole substance approach.

Due to the nature of the testing material (the whole substance as manufactured containing all its con- 
stituents), the performance of (eco)toxicological studies is in many cases not possible. In such specific cases, the constituent or block of constituents for which the hazard assessment of the original source was critical can be used for them. According to this, if it can be shown that constituents belonging to one block are of no eco(toxicological) concern, it can be concluded that the whole examined block does not affect the hazard profile and equivalence can be granted. Further detailed information on testing of UVCB substance as indicated by ECHA are given in REACH guidance Chapters R.7 Endpoint specific guidance and R.11: PBT/vPvB assessment and EFEO/IFRA guidelines $^{14}$.

\section{Technical Equivalence of Technical Grade Active Ingredients of Microorganism}

In the recent years, the use of biocontrol agents has become very popular. One of the most popular classes of biocontrol agents are microorganisms, which includes bacteria, entomopathogenic fungi, and viruses. For the purpose of the evaluation of technical equivalence for identical microorganism strains or isolates approved under Regulation (EC) 1107/2009, the European Commission published at the end of 2014 a new version of the SANCO guideline: SANCO/12823/2012 -rev. 4 (12 December 2014). ${ }^{15}$ This Guidance Document is applicable for changes to the same strain of a microorganism but only in the framework of application for authorisation of plant protection products at Member State level. The purpose of this SANCO document is to provide guidance for the assessment of technical equivalence of microorganisms used in plant protection products.

Similar to the evaluation of technical equivalence on chemical active substances, also here different scenarios are considered, as for example change of location of a manufacturing plant, scaling up the fermentation vessel and change of manufacturing process (equipment, reaction conditions, or raw materials). Furthermore, as indicated by ECHA in the Guidance of active microorganisms and biocidal products, once production of a microorganism active substance changes from a pilot plant production system to an industrial scale, an application for technical equivalence assessment has to be filed to ECHA in the case of Biocidal products. ${ }^{16}$
Also for assessing the technical equivalence of microorganism used as active substances, a two-tiered approach applies. Both sources of the microorganism are considered equivalent after Tier 1 evaluation, when:

- the content of the active microorganism in the new source is higher than or equal to the reference source

- the composition of materials used for the production is similar,

- the content of relevant metabolites/toxins and contaminants in the new source is lower or equal than in the reference source.

If the afore-mentioned Tier I criteria are not fulfilled, an assessment of the potential hazard to human health and the environment shall be performed, and ie a Tier II assessment is required.

\section{Technical Equivalence of In Situ Generated Active Substances under BPR}

During the 59th meeting of representatives of Members States Competent Authorities for the implementation of the BPR, the management of in situ generated active substances was discussed. The outcome of the meeting was published by the European Commission in the CA-March15-Doc.5.1-Final; Revised on 23 June $2015^{17}$. It was acknowledged that a comparison of the chemical composition and hazard profile of the in situ generated active substances is technically difficult, and sometime not possible, since it is very challenging to establish a reference source. As recommended, the technical specifications can be es-

14 EFEO/IFRA, 'EFEO/IFRA Guidelines on Substance identification and Sameness of Natural Complex Substances (NCS) under REACH and CLP' (5 August 2015) <http://www.ifraorg.org/view _document.aspx?docld=23628 > accessed 12 February 2019 .

15 Guidance Document for the Assessment of the Equivalence of Technical Grade Active Ingredients Foridentical Microbial Strains or Isolates Approved under Regulation (EC) No 1107/2009.

16 ECHA, 'Guidance on BPR: Volume V. Guidance on Active MicroOrganisms and Biocidal Products Version 2.1 (March 2017) $<$ https://echa.europa.eu/documents/10162/23036412/biocides guidance_micro_organisms_en.pdf/4d028d38-6d3c-4f2d-80f7 -3aa2118ca49a> accessed 12 February 2019.

17 Guidance to specify information requirements for in situ generated free radicals for substance approval in the context of the BPR (23 June 2015). 
tablished either for the active substance itself or for its precursors, as appropriate, at the time of the active substance approval. Additionally, a reference to the existing standards, such as CEN standards, ${ }^{18}$ is also possible. Consequently, the applicant needs to demonstrate at the time of product authorisation that the precursors or the active substances meet the agreed specifications. Finally it was concluded, that when an in situ generated active substance may also be placed on the market as standalone active substance (eg peracetic acid), specifications would still have to be established in order to allow for the establishment of a technical equivalence.

\section{Miscellaneous}

EFSA published in 2017 an EFSA Technical Report on the outcome of the pesticides peer review meeting on general recurring issues in physical and chemical properties and analytical methods under Regulation (EC) No 1107/2009. ${ }^{19}$ In this report, EFSA and the Member States' Authorities discuss different issues related to the assessment of the impurity profiles, the proposed specifications, reference specification for the multiple sources, etc., and provide some recommendations at the end. The examples presented and the outcome show the importance of the correct interpretation of the different scenarios and situations referring to impurity profiles and minimum purities set in the reference source.

In one of the discussed issues, EFSA refers for example to the EFSA Technical Report on the outcome of the pesticides peer review meeting on general recurring issues in mammalian toxicology. ${ }^{20}$ There it is stated that the guidance document on the assessment of the equivalence of technical materials can be used in the evaluation of active substance in the renewal process. This means that the SANCO/10597/2003 - rev. 10.1 Guidance can possibly be

18 European Committee for Standardisation (CEN).

19 EFSA, 'Outcome of the Pesticides Peer Review Meeting on General Recurring Issues in Physical and Chemical Properties and Analytical Methods European Food Safety Authority' (2 May 2017) <https://www.efsa.europa.eu/en/supporting/pub/en-1221> accessed 12 February.

20 EFSA, 'Outcome of the Pesticides Peer Review Meeting on General Recurring Issues in Mammalian Toxicology European Food Safety Authority' (25 July 2016) <https://www.efsa.europa.eu/en/ supporting/pub/en-1485> accessed 12 February 2019. used for two purposes: once for setting the specifications during the approval and second in the next step when assessing technical equivalence of different technical materials. The following example is given: specification for a certain impurity can be set at a $50 \%$ higher level than found in toxicological/ecotoxicological batches and subsequently a further $50 \%$ deviation from the toxicological/ecotoxicological batch level would be allowed when assessing technical equivalence. As an outcome of the discussion, Member States indicated misuse of the equivalence guidance by using this guidance to set the reference specification and then again for equivalence check. EFSA considered misinterpretation of the cited document since the guidance document on equivalence, comparing specifications, should not be used in the process of assessing whether the proposed specification is supported or not by manufacturing data. Its use in the toxicological evaluation is aimed solely to assess whether the proposed specification is covered by toxicological data.

Another issue discussed during that meeting and presented in the resulting Technical Report refers to relevant impurity - reference specification. One of the Member States shared the details of an application in which the new specification was proposed which led to an increase of the value for a genotoxic impurity by three times in comparison to the reference specifications. Since guidance document on the assessment of equivalence does not allow for equivalence if the new source has more harmful effects due to its impurities compared to the reference source, two options were discussed: either setting a new specification or prepare a new equivalence assessment. As an outcome of the discussion, an equivalence assessment (containing an appropriate justification and alternatively QC data) against the old reference specification was proposed. Additionally, EFSA noted that in many cases except from the minimum purity of the active substance, impurities and toxicological data are not known from the old batches, as these data was not a requirement in the past. Consequently it was acknowledged, that setting the reference specification is a risk-management' decision.

\section{Conclusion}

Globally acting chemical companies have very often more than one manufacturing site for the production 
active substances or technical materials, and manufacturing processes of the active substance need sometimes to be adjusted. Thus, the assessment of the technical equivalence is critical to ensure that the chemical compositions and hazard profiles of different technical materials remain equivalent to the original reference specification. The detailed analysis of technical materials originating from a new or additional source, in the scope of a two-tiered approach, allows for identifying any additional or more severe hazard effect on human health and the environment. Technical Equivalence procedure provides the formulators of products, the European consumers and the environment with the necessary safety regarding the quality of the active substances put on the EU market.
It is quite common that the same active substance is used for both, crop protection and biocidal products. Thus, this article covers the two main Regulations Reg. 1107/2009 and Reg. 528/2012 portraying the similar principles when assessing technical equivalence of active substances' and its sources.

The procedures described in the guidance documents published by the European Commission and European Chemical Agency are relatively clear and detailed, allowing applicants to prepare a smooth application for technical equivalence. Difficulties in the process are related to the confidential provisions of composition details, to prevent third-party applicants to know what are the exact limits defined in the reference source(s), making it difficult to match a specification of unknown composition. 